\title{
MOTIVACIÓN LECTORA INICIAL EN \\ CHILE: UN ESTUDIO EXPLORATORIO
}

\author{
READING MOTIVATION IN CHILE: \\ AN EXPLORATORY STUDY IN THE FIRST \\ YEARS OF ELEMENTARY EDUCATION
}

Pelusa Orellana García

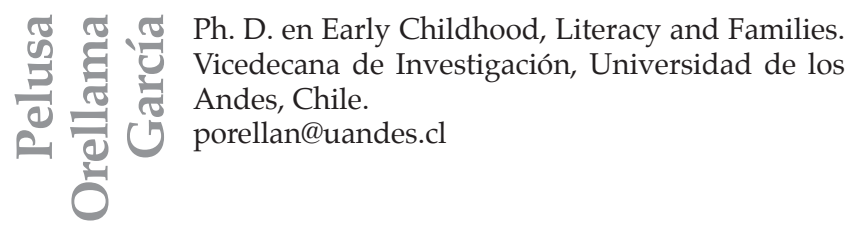

\section{RESUMEN}

Este estudio analiza el desarrollo de la motivación lectora en estudiantes chilenos que se inician en el proceso lector. Usando la versión en español de la Encuesta Yo y Mi Perfil Lector, desarrollada por Marinak, Malloy, Gambrell y Mazzoni en 2015, evaluamos a 302 estudiantes al inicio y final del año escolar. Los resultados muestran un descenso en la motivación para los niños de primer y segundo grado hacia el fin de año, un incremento en Kínder, y diferencias significativas entre niños y niñas. Estos resultados permiten conocer en mayor profundidad el papel que juega la motivación en el desarrollo lector, en especial en niños pequeños de países latinoamericanos, donde la evidencia empírica 
es prácticamente inexistente, y considerando la estrecha relación entre dimensiones afectivas y desempeño lector posterior.

Palabras clave: motivación lectora, lectura inicial, autoconcepto, valoración de la lectura.

\begin{abstract}
The current study analyzes the development of reading motivation among Chilean beginning readers. Using the Spanish version of Me and My Reading Profile Survey developed by Marinak, Malloy, Gambrell, and Mazzoni in 2015, we assessed 302 Kíndergarten through secondgrade students at the beginning and end of the school year. Results show a decrease in reading motivation for children in first and second grade, an increase in Kíndergarten students, and significant differences among boys and girls. These findings allow us to better understand the role of reading motivation in reading acquisition, particularly among beginning readers, where empirical evidence for Latin American countries is almost non-existent, and especially in relation to the strong connection between motivation to read and reading achievement in the upper grades.
\end{abstract}

Key words: reading motivation, beginning readers, self-concept, value of reading.

\title{
INTRODUCCIÓN
}

Desarrollar habilidades de comprensión lectora es un requisito fundamental para el éxito escolar, y una de las principales tareas de los primeros años de escolaridad. Una buena comprensión lectora es necesaria para avanzar en el sistema escolar, pero también para funcionar adecuadamente en la sociedad, desarrollando con autonomía tareas cotidianas, cumpliendo deberes cívicos, e insertándose en el campo laboral de manera competente. La denominada alfabetización funcional se refiere precisamente a la capacidad de comprender textos y utilizarlos para llevar a cabo las tareas del diario vivir (OECD, 2000; Collins 
y O'Brien, 2003). Es sabido que, a nivel mundial, el desempeño de los países latinoamericanos en pruebas de alfabetización funcional se encuentra aún por debajo del promedio de los países de la OECD. Ello se desprende de análisis de resultados en pruebas PISA, PIRLS, SERCE, y TERCE, en los que, pese a que se observan algunas mejoras importantes en la última década, nuestro desempeño está aún lejos de lo esperable para contextos de alto procesamiento de información escrita.

Por otra parte, en los últimos 30 años ha crecido el interés por relacionar aspectos afectivos de la lectura al desempeño lector. Teorías como la de la autodeterminación (Ryan y Deci, 2000; Gagné y Deci, 2005), que distingue entre motivación intrínseca y extrínseca, o la teoría de expectativa-valor (Wigfield y Eccles, 2000; Cantrell, Rintamaa, Anderman y Anderman, 2017) han intentado explicar esta relación. Algunos estudios documentan esta correlación (e.g. Wigfield, Gladstone y Turci, 2016; McKenna, Kear, Elsworth, 1995), planteando que cuando el entorno de las actividades lectoras es estimulante, los alumnos mejoran su lectura. Dentro de las características de un ambiente que suscita el interés por la lectura están: la libertad de elección, el tiempo para leer en forma recreativa, el acceso a libros en el aula, y la promoción de espacios para que los estudiantes comenten sus lecturas. Los profesores que promueven actitudes favorables hacia la lectura son también capaces de transmitirlo a sus estudiantes.

En Chile, la relación entre la motivación lectora y la comprensión se ha estudiado principalmente en niños mayores de 9 años. Los datos señalan que en general más del 50\% de los chilenos entre 18 y 60 años se declara no lector (Fundación La Fuente/ Adimark, 2011), y los resultados de la evaluación PIRLS 2016 indican una estrecha relación entre motivación lectora y comprensión para estudiantes de 9 años (Agencia de la Calidad de la Educación, 2017). Asimismo, la mayor motivación por la lectura en estudiantes chilenos se asocia al acceso a libros y los hábitos lectores de los padres. Desafortunadamente, para los estudiantes chilenos sólo el $27 \%$ de los padres muestran afición a la lectura, lo que se encuentra por debajo del promedio mundial. Estos resultados avalan la importancia y la necesidad de estudiar la evolución de la motivación lectora, su relación con la comprensión, y las diferencias que se originan a partir de aspectos como curso o género de los estudiantes. 


\section{REVISIÓN DE LA LITERATURA}

\section{Motivación a la lectura, su evolución e importancia}

El estudio de la motivación lectora se remonta al inicio del siglo 20, cuando la psicología de la lectura comenzó a ser estudiada con mayor interés. Con el paso de los años se ha reforzado la idea de que la motivación lectora es un factor determinante en la comprensión, durante prácticamente toda la vida (Afflerbach, Cho, Kim, Crassas, y Doyle, 2015; Conradi, Jang, y McKenna, 2014; Schwanenflugel y Knapp, 2015). Ello ha hecho que la investigación dirija su atención a fenómenos tales como motivación intrínseca vs. extrínseca, diferencias en motivación a nivel intercultural, según edad, sexo, nivel socioeconómico, y género literario, entre otros. No sorprende, por tanto, que los grandes estudios mundiales como PISA, PIRLS, SERCE y TERCE consideren la variable motivacional a la hora de analizar los resultados en el desempeño lector de los estudiantes.

Comprender la importancia de los factores afectivos en la lectura tiene varias aristas. Por una parte, quienes se motivan más por leer tienden a dedicarle más tiempo, y como consecuencia, comprenden mejor lo que leen (Allington, 2014). Los resultados de las evaluaciones realizadas por la OECD, a lo largo de los últimos 10 años, dan cuenta de una importante correlación entre niveles de comprensión lectora y motivación para los estudiantes de 15 años a nivel mundial (OECD, 2010), pero esta correlación también ha sido observada en los últimos años de enseñanza primaria, estudiantes universitarios, e incluso adultos (Schwanenflugel y Knapp, 2015). De hecho, la varianza en el desempeño lector - que se explica por la cantidad de tiempo que las personas dedican a la lectura- se incrementa de un $12 \%$ en los primeros años de escolaridad, a un 34\% en la época universitaria (Mol y Bus, 2011). Por otra parte, los estudios de motivación lectora buscan además comprender aspectos cualitativos de la lectura de quienes están más motivados por ella, respecto de quienes no se motivan. Componentes tales como el autoconcepto lector, el valor social de la lectura, las expectativas de los lectores, la libertad de elección a la hora de leer, y los tipos de lectura que se realizan son algunos de los factores que ayudan a comprender cómo se desarrolla la motivación e interés por la lectura a distintas 
edades. Por ejemplo, las personas con mayores niveles de motivación, suelen tener una percepción de sus habilidades lectoras superior a la de quienes no se sienten motivados (Guthrie y Wigfield, 2000).

Numerosos estudios demuestran que la motivación lectora — pese a ser bastante alta en los niños más pequeños-, suele disminuir considerablemente a medida que los estudiantes avanzan en la escuela primaria (McKenna, et al., 1995). Las bajas más importantes se dan al inicio del quinto o sexto grado, y al ingresar a la enseñanza media (noveno grado) (McKenna, Conradi, Lawrence, Jang, y Meyer, 2012; Lepper, Corpus, y Iyengar, 2005). En países como Finlandia y Singapur, por ejemplo, la correlación entre motivación y habilidad de comprensión en los estudiantes de 15 años solía ser muy alta; sin embargo, en las últimas evaluaciones PISA, se ha observado un descenso similar al que se constata en países con menos puntaje promedio en comprensión. Algunos sugieren que la baja global en el interés por la lectura está asociado a la irrupción de la lectura digital y online; y que más que una desmotivación por leer, lo que se observa es un cambio en el contexto en el cual se lee (Maynard, 2010; Larson, 2010). La evidencia al respecto no es contundente y se requiere de más investigación acerca de este fenómeno.

\section{Modelos teóricos subyacentes}

Dos teorías prevalentes respecto de la motivación a la lectura son las teorías de la autodeterminación y de la expectativa-valor. Ambas teorías pertenecen a la categoría de teorías acerca de creencias, valores y metas, y establecen que los procesos que influyen en la motivación en las personas pueden controlarse por ellos mismos (Arango, 2018). En pocas palabras, estas teorías se refieren a las creencias que nosotros mismos tenemos respecto a nuestra capacidad de alcanzar una meta, factores afectivos que podrían determinar nuestra capacidad de realizar tareas más desafiantes, y niveles de autoeficacia en general.

La teoría de la autodeterminación fue propuesta por Deci y Ryan (2000) y plantea que los individuos poseen tres necesidades básicas: un deseo inherente de ser competentes, de sentirse capaces de lograr 
lo que quieren alcanzar, la necesidad de autonomía y la necesidad de relacionarse con los demás. Según Deci y Ryan, éstas son las necesidades que impulsan la motivación intrínseca de las personas, así como también su comportamiento autodeterminado (Deci y Ryan, 2002). Aplicado al ámbito de la lectura, este planteamiento teórico promueve un autoconcepto de lector favorable como factor necesario para desarrollar mayor motivación lectora, así como también una valoración positiva de la lectura como actividad humana. La teoría de expectativa-valor establece que la elección, persistencia y desempeño de una persona en una determinada tarea se explica por sus creencias acerca de qué tan bien pueden realizar la tarea y cuánto la valoran (Wigfield y Eccles, 2000). Desde este sustento teórico, los lectores motivados tienen una percepción positiva de su capacidad de leer un determinado texto, y valoran la lectura al igual que en la teoría de la autodeterminación. En ambos postulados teóricos, la motivación a la lectura es entendida como intrínseca; es decir, son creencias, valores, afectos y motivaciones que surgen del individuo - y no de su entorno- los que lo llevan a una mayor motivación por leer.

El Perfil Yo y Mi Lectura se fundamenta en estos dos planteamientos teóricos. Se construyó sobre la base de dos constructos que, si bien se correlacionan, responden a estos dos aspectos de la lectura: las creencias sobre la propia capacidad de leer, la autonomía y la capacidad de enfrentar desafíos, por un lado, y por el otro la valoración de la lectura como una actividad en la que el individuo se relaciona con otros.

\section{Diferencias entre niños y niñas}

Diversos estudios han analizado las diferencias en los intereses y hábitos lectores de niños y niñas alrededor del mundo, así como también respecto de su desempeño lector. Las diferencias entre niños y niñas se pueden observar en prácticamente todos los países en evaluaciones como PIRLS 2010 (Mullis, Martin, Foy, y Drucker, 2012), como también en PISA con estudiantes mayores (Brozo, Sulkunen, Shiel, Garbe, Pandian, y Valtin, 2014), e incluso a partir de tercer grado (Gambrell, Palmer, Codling y Mazzoni, 1996). Por ejemplo, si bien niños y niñas tienen un nivel similar de auto concepto lector, los varones de 
tercer grado valoran menos la lectura que las niñas (Marinak y Gambrell, 2007, 2010; Brozo et al, 2014). Por otra parte, las diferencias entre niños y niñas han sido explicadas por aspectos evolutivos, como por ejemplo el hecho de que las mujeres desarrollan el lenguaje antes que los varones. Gurian y Henley (2001) indican que casi el 80\% de los estudiantes no motivados por la lectura son hombres. Asimismo, desde un punto de vista sociocultural, las diferencias en motivación lectora se han explicado en función de visiones estereotipadas en las que la lectura, en muchas culturas, es percibida como una actividad eminentemente femenina.

En Chile, las diferencias en motivación y desempeño lector han tenido siempre un sesgo favorable a las mujeres. Esta diferencia se observa como una constante a lo largo del sistema escolar, independiente además del nivel socioeconómico de los estudiantes. Un estudio de Orellana y Baldwin (2017) confirmó esta tendencia en niños chilenos de tercero a sexto grado, tanto para motivación como para la habilidad de comprensión lectora. Los resultados de este estudio son coincidentes con investigaciones de mayor escala en el país (Agencia de la Calidad en Educación, 2015; 2017), lo que hace suponer que esta tendencia se ha mantenido en el tiempo.

\section{METODOLOGÍA}

\section{Diseño y participantes}

El presente estudio es exploratorio en su naturaleza, pues intenta responder las siguientes preguntas: ¿cómo evoluciona la motivación a la lectura en los estudiantes chilenos que inician su proceso lector?, ¿existen diferencias en la trayectoria de la motivación lectora según curso y sexo?

Como mencionamos anteriormente, no existen estudios de motivación lectora en los primeros años de enseñanza primaria en Chile, y dado que en estudios de gran escala (como TERCE, PIRLS y PISA) se muestra una importante asociación entre motivación y comprensión en los cursos superiores, se consideró esencial analizar cómo se desarrolla la motivación a la lectura al inicio de la escolaridad. 
Un total de 302 estudiantes chilenos de Kínder, primero y segundo año de primaria participaron en este estudio. De ese total, 99 cursaban Kínder, 84 primero, y 119 segundo año de primaria. Un $49.3 \%$ de los participantes correspondía a varones. La muestra proviene de dos establecimientos educacionales de un distrito metropolitano de educación. Uno de los colegios era privado y el otro particular subvencionado; es decir, recibía algún tipo de apoyo financiero de parte del Estado. Los alumnos del colegio privado provenían de hogares de nivel socioeconómico alto, mientras que los provenientes del colegio subvencionado correspondían a un nivel de ingreso medio. En términos de distribución, el 51\% de los evaluados pertenecía al establecimiento subvencionado. No fue posible reclutar estudiantes de colegios municipalizados, que suelen recibir a familias de nivel socioeconómico bajo y medio-bajo.

\section{Instrumento}

La Encuesta MMRP consta de 18 ítems para evaluar la motivación lectora. De esos 18 ítems, 9 se relacionan con el constructo de autoconcepto lector y 9 con el de valoración de la lectura. El instrumento original se desarrolló en idioma inglés y, para el presente estudio, fue traducido usando el método del back- translation (Harkness y Schoua-Glusberg, 1998). El procedimiento del back-translation consiste en traducir primero del idioma primario al secundario, luego volver a traducir al idioma original, en tercer lugar, realizar una validación por parte de seis expertos (docentes de educación inicial y primaria), y, finalmente, elaborar la traducción definitiva al segundo idioma. En la primera traducción se recurrió a un hablante nativo de español bilingüe en inglés, mientras que en la segunda traducción, otro hablante nativo del español realizó la versión definitiva. El objetivo de este tipo de traducciones es lograr una equivalencia conceptual en los documentos, más que una traducción literal. Posteriormente, se realizó un juicio de expertos en el que seis evaluadoras y maestras de enseñanza primaria revisaron cada ítem para asegurar su adecuación al contexto lingüístico y cultural chileno, y para verificar que no existiera ningún tipo de sesgo. La versión final del instrumento se piloteó con una muestra pequeña de estudiantes 
para asegurar que no hubiese sesgos culturales ni terminología poco clara para niños tan pequeños.

Cada ítem de la encuesta está construido sobre la base de una escala Likert de 3 puntos, y los alumnos deben responder una pregunta sencilla, marcando una de las tres alternativas de la escala. Se escogió este tipo de escala considerando la evidencia que apunta a que, para niños tan pequeños, la discriminación entre más de 3 alternativas suele ser dificultosa (Case y Khanna, 1981; Nitko, 1983). El alfa de Cronbach para la primera aplicación de la encuesta dio un coeficiente de confiabilidad de .822 .

\section{Procedimientos}

La aplicación del MMRP se administró dos veces en el año escolar, una al comienzo (abril) y otra hacia el final (noviembre). Para ello se obtuvo primeramente la autorización en cada establecimiento y los consentimientos informados de parte de los padres de cada uno de los estudiantes. La aplicación fue realizada en formato de lápiz y papel, por los docentes, a quienes se les realizó una breve capacitación. Los docentes explicaron a los estudiantes cómo responder la encuesta. En el caso de los alumnos de Kínder, los docentes lo administraron en grupos pequeños de 5 alumnos, mientras que en los cursos superiores se realizó con el grupo completo. La encuesta contiene dos ítems de práctica, los cuales fueron respondidos por los estudiantes antes de iniciar la evaluación, a fin de familiarizarse con el formato del instrumento. Como se muestra en el anexo, cada ítem está asociado a un dibujo, lo que facilita que los niños que aún no leen en forma independiente puedan seguir al docente fácilmente. Así, los maestros entregaban la siguiente instrucción previamente a responder cada ítem: «Pon tu dedo en la figura del oso [correspondiente al ítem 1]». Luego, los maestros se aseguraban de que todos los niños estuvieran en el mismo ítem antes de leer el enunciado del ítem y las tres alternativas de la escala Likert. A los alumnos se les indicó que pensaran cuidadosamente su respuesta y marcaran la que más se parecía a cómo se sentían ellos respecto de la lectura. Finalmente, los maestros debían esperar que todos los 
alumnos hubieran respondido el ítem antes de pasar al siguiente. La administración de la MMRP tuvo una duración de entre 15 y 20 minutos por curso.

\section{Análisis}

En primer lugar, se realizaron análisis preliminares para examinar el comportamiento de los datos. Luego, calculamos la consistencia interna usando el método de alfa de Cronbach, para la MMRP en su totalidad, y luego para cada sub escala (autoconcepto de lector y valoración de la lectura).

En segundo lugar, se compararon las medias de los grupos a inicio y final del año escolar, con el fin de responder a la pregunta de si existen diferencias significativas entre la motivación lectora entre esos dos períodos, si se observan diferencias por curso y entre niños y niñas. Para ello se utilizaron pruebas t y análisis de varianza. Finalmente, se analizaron las correlaciones entre los constructos de autoconcepto lector y valoración de la lectura en cada curso y según sexo, con el fin de determinar el peso de cada constructo y las potenciales diferencias entre los grupos etarios y entre niños y niñas.

\section{RESULTADOS}

\section{Análisis preliminares}

En la tabla 1 presentamos las estadísticas descriptivas de ambas mediciones. Los valores totales de la MMRP se incrementan a medida que aumenta el curso de los estudiantes al inicio del año escolar, mostrando que los alumnos de segundo grado poseen un mayor nivel de motivación a la lectura que sus pares de primer grado y Kínder. A fines del año escolar, sin embargo, este resultado se invierte, observándose menor motivación lectora en los alumnos de segundo respecto del inicio del año anterior. Los alumnos de segundo grado a fines del año, son también los que obtienen el valor más bajo en el total de la encuesta. 
Tabla 1. Estadísticas descriptivas por curso

\begin{tabular}{lcccccc} 
Curso & \multicolumn{3}{c}{ Inicio } & \multicolumn{3}{c}{ Fin } \\
\hline Kínder & $\mathrm{M}$ & (DS) & Rango & $\mathrm{M}$ & (DS) & Rango \\
\hline Primero & 34.7 & 10.9 & $27-31$ & 35.2 & 2.6 & $24-42$ \\
\hline Segundo & 36.1 & 6.33 & $11-54$ & 35.4 & 2.3 & $31-45$ \\
\hline
\end{tabular}

En la tabla 2 se presentan los resultados desagregados por constructo. En general, los promedios obtenidos en ambos constructos son bastante similares y se encuentran en un rango que va de los 16.4 a los 19.1 puntos en promedio. El autoconcepto disminuye para los tres grupos etarios desde el inicio hacia el fin de año, y lo mismo ocurre para la valoración lectora, salvo en Kínder donde aumenta al finalizar el año. La magnitud de la disminución es pequeña salvo en segundo grado donde el autoconcepto disminuye 2 puntos y la valoración casi 3 puntos. El único incremento, observado en valoración lectora, tiene una magnitud inferior a un punto (.7).

Tabla 2. Estadísticas descriptivas según constructo

\begin{tabular}{lllll} 
Curso & \multicolumn{2}{c}{ Autoconcepto } & \multicolumn{2}{c}{ Valoración } \\
& M (Inicio) & M (Fin) & M (Inicio) & M (Fin) \\
& DS & DS & DS & DS \\
& Rango & Rango & Rango & Rango \\
\hline Kínder & 17.6 & 17.4 & 17.1 & 17.8 \\
& 2.2 & 1.8 & 2.3 & 2.8 \\
& $12-23$ & $13-22$ & $12-32$ & $11-21$ \\
\hline Primero & 17.5 & 17.2 & 18.6 & 18.3 \\
& 3.5 & 1.8 & 3.2 & 1.5 \\
& $4-27$ & $13-23$ & $7-27$ & $14-23$ \\
\hline Segundo & 18.6 & 16.6 & 19.1 & 16.4 \\
& 3.2 & 3.1 & 3.3 & 3.5 \\
& $13-27$ & $9-27$ & $13-27$ & $9-27$ \\
\hline
\end{tabular}




\section{Análisis principales}

Se realizó una prueba de muestras pareadas para comparar la motivación lectora al inicio $(\mathrm{M}=36.3, \mathrm{SD}=5.5)$ y final del año escolar $(\mathrm{M}=$ 34.4, $\mathrm{SD}=4.47)$. La diferencia entre los puntajes de ambos períodos resultó ser estadísticamente significativa $(t=4.669, \mathrm{p}<.000)$. Para desagregar estos resultados y determinar si las diferencias entre inicio y año escolar en cada curso diferían, se realizó un análisis de varianza. Los resultados muestran que las diferencias entre los grupos, en ambos períodos sí son significativas. Para el inicio del año escolar el valor fue $\mathrm{F}(2,299)=7.832, p=.00$ y para fines de año $\mathrm{F}(2,299)=9.762, p=.000$.

Al desagregar estos resultados por constructo, los resultados del análisis de varianza también mostraron diferencias estadísticamente significativas entre el inicio del año escolar tanto para autoconcepto lector como para valoración de la lectura. La tabla 3 muestra los resultados de estos análisis.

Tabla 3. Análisis de varianza por curso y constructo

\begin{tabular}{lcccc}
\hline Fuente & $d f$ & $M S$ & $F$ & $p$ \\
\hline $\begin{array}{l}\text { Inicio año escolar } \\
\text { Motivación }\end{array}$ & 2 & 29.49 & 7.832 & .000 \\
Autoconcepto & 2 & 9.188 & 4.013 & .019 \\
Valoración & 2 & 8.955 & 11.77 & .000 \\
\hline
\end{tabular}

Fin año escolar

\begin{tabular}{lcccc} 
Motivación & 2 & 19.001 & 9.762 & .000 \\
Autoconcepto & 2 & 5.943 & 3.272 & .039 \\
Valoración & 2 & 6.299 & 14.59 & .000 \\
\hline
\end{tabular}


Un segundo análisis revisó las potenciales diferencias según sexo, tanto para la motivación lectora en general como según constructo. Las diferencias no son estadísticamente significativas para la motivación en general ni para el autoconcepto lector o valoración de la lectura por separado. Si se desagregan por curso estas diferencias son significativas en Kíndergarten al inicio del año, tanto para la motivación lectora en general como para el autoconcepto, pero no para la valoración ni para ninguna de las variables al finalizar el año escolar. En primer grado ninguna diferencia resultó ser significativa. En segundo grado la única diferencia significativa se observó en el autoconcepto lector hacia fines del año escolar.

Finalmente, las correlaciones entre autoconcepto y valoración de la lectura son más altas para las niñas $\left(.655^{* *}\right)$ que para los varones $\left(.590^{* *}\right)$. Si se analizan por curso, la correlación entre ambos constructos es mayor para los niños $\left(.702^{* *}\right)$ en primer grado que para las niñas $\left(.656^{* *}\right)$; en segundo grado es mayor para las mujeres $\left(.842^{* *}\right)$. No se observaron correlaciones importantes en Kínder.

\section{Discusión}

En este estudio examinamos la evolución de la motivación lectora en niños que recién comienzan su proceso lector. Desde la teoría de la autodeterminación, evaluamos la motivación lectora a partir de los constructos de autoconcepto y valoración, al inicio y fin del año escolar. Los resultados muestran un descenso leve del autoconcepto lector de los alumnos de Kínder, primer y segundo grado, y un descenso más abrupto en la valoración lectora, en particular en segundo grado. El autoconcepto en los estudiantes de Kínder es superior al finalizar el año escolar. Estos resultados muestran una tendencia similar a la observada en estudios previos con estudiantes en los cursos superiores de la educación primaria (McKenna et al, 2012; Kush y Watkins, 1996; Durik, Vida y Eccles, 2006). El descenso en la motivación, tanto desde el autoconcepto como desde la valoración lectora, puede explicarse desde distintos puntos de vista, como detallaremos a continuación.

Tradicionalmente, los modelos teóricos han establecido dos grandes momentos en la adquisición de la lectura (Chall y Jacobs, 2003). 
El primero se ha denominado «aprender a leer», va desde Kíndergarten a tercero básico, y se centra principalmente en la adquisición de destrezas para decodificar el texto y, a partir de dicha decodificación, acceder al significado. En este momento se espera que los alumnos adquieran un cierto grado de automaticidad en algunos subprocesos lectores (identificación de letras y palabras de uso frecuente, velocidad y acuciosidad lectora), para dedicar una mayor carga cognitiva a tareas de construcción de significado. El segundo momento se ha denominado «leer para aprender» y se inicia en el cuarto grado. Se espera que los estudiantes - habiendo ya adquirido la necesaria automaticidad en ciertos subprocesos lectores-, puedan dedicar toda su atención a subprocesos más complejos como son la comprensión, el vocabulario, y en particular la lectura crítica e inferencial. Esta división ha sido cuestionada en el último tiempo argumentándose que el aprender a leer y el leer para aprender suelen ocurrir de manera más bien paralela (Robb, 2011). Los lectores usan lo que saben y conocen para mejorar su aprendizaje lector más allá del tercer grado, y de la misma manera, aprenden mientras leen, aun cuando los subprocesos lectores están en desarrollo. Pese a lo anterior, en muchas aulas de clase prevalece aún esta mirada en que la comprensión lectora se trabaja muy poco en los cursos de primer a tercer grado, y el énfasis en los subprocesos tendientes a la adquisición de la automaticidad, sin el componente de construcción de significado puede acarrear un desinterés por la lectura por varias razones. La primera es que los niños rápidamente se dan cuenta de sus propias capacidades y se etiquetan como «buenos» $\mathrm{o}$ «malos» lectores, no en función de sus hábitos o de su nivel de comprensión, sino más bien en función de su velocidad lectora. Muchas veces, al aplicar el Perfil Yo y Mi Lectura en forma oral y preguntar a los niños en Chile si se consideran buenos o malos lectores, la respuesta ha sido: «soy bueno, leo 120» (refiriéndose a que leen 120 palabras por minuto). De esta manera, el entusiasmo inicial por la lectura, que se origina en Kínder, cuando los niños comienzan a visitar la biblioteca y a elegir libremente lo que quieren leer, disminuye a medida que avanzan en el sistema escolar y el foco cambia del disfrute a la evaluación. En ese momento, los alumnos son absolutamente conscientes de quién es el «buen» lector en función de la velocidad, y rápidamente se desmotivan si su lectura no es fluida, lo que es absolutamente esperable en ese nivel. La asociación entre lectura y 
velocidad lectora ha sido siempre perjudicial para mantener el interés por la lectura en los estudiantes a lo largo de sus vidas (Morgan, Fuchs, Compton, Cordray, y Fuchs, 2008; Ludwig, 2007).

Un segundo factor tiene que ver con la obligatoriedad de la lectura. Como mencionamos anteriormente, la lectura en Kínder suele ser bastante libre en el sentido de que los estudiantes pueden acceder a libros de una biblioteca generalmente atractiva. Asimismo, las maestras suelen realizar actividades de lectura grupal en la que normalmente cuentan a los niños un cuento ilustrado de su interés, y, si se trabaja desde la comprensión oral, generalmente mantiene la atención de los niños.

A medida que avanzan en el sistema escolar y los alumnos aprenden a decodificar, la lectura suele transformarse en una actividad tediosa de lectura repetitiva de textos sin mucho sentido. Pocas veces se leen cuentos en voz alta, o se invita a los alumnos a explorar libremente los libros en la sala. En muchas ocasiones se pide a los alumnos trabajar la lectura en voz alta en la casa, o directamente leer frente al curso, lo que suele causar frustración a quienes se reconocen como «malos lectores» desde la perspectiva de la fluidez. Como consecuencia de ello, la motivación por la lectura como actividad escogida para el tiempo libre, disminuye de manera importante. Es posible, que, en el caso de los alumnos evaluados en el presente estudio, las bajas en autoconcepto lector de Kínder a segundo grado, y desde el inicio al fin del año escolar tengan que ver con estos dos factores.

Un tercer factor es la valoración social de la lectura, y que, en los alumnos evaluados, disminuye especialmente hacia fines de segundo grado. Si bien en el presente estudio no se identificaron diferencias significativas según sexo, la literatura establece que son mayoritariamente los hombres quienes valoran menos la lectura a lo largo de la vida escolar y adulta. Ello suele atribuirse a la prevalencia de modelos lectores femeninos en la escuela y en la casa, lo que podría incidir en una valoración negativa. 


\section{CONCLUSIÓN Y LIMITACIONES}

El presente estudio muestra que la motivación lectora en niños chilenos que se inician en la lectura varía de curso en curso, incrementándose en Kíndergarten, y disminuyendo en primer y segundo grado. Los constructos de autoconcepto lector y valoración de la lectura evolucionan de manera distinta en cada curso, siendo mayor la disminución de la valoración de la lectura, en particular en segundo grado. Las diferencias de género no son significativas en este grupo etario, pero la evidencia a nivel nacional muestra un sesgo a favor de las mujeres a lo largo de la etapa escolar. Las implicancias pedagógicas de estos hallazgos son importantes por cuanto podrían ayudar a los docentes a tener en cuenta la evolución de la motivación lectora durante el año escolar y a lo largo de la escolaridad, considerando además su relación con el perfeccionamiento de las habilidades de comprensión.

Conocer estas implicaciones (tener un buen o mal autoconcepto lector o valoración de la lectura) podría orientar a los docentes a crear espacios para fomentar la elección de libros, valorar instancias de discusión, o tener en cuenta las preferencias lectoras de uno y otro sexo. Este tipo de acciones permitiría mantener mayores niveles de autoconcepto y valoración a lo largo de toda la etapa escolar.

Como en todo estudio exploratorio, el presente tiene algunas limitaciones importantes de considerar. La primera es el tamaño de la muestra, y el hecho de que en ésta no se incluyeron estudiantes provenientes de colegios municipalizados, donde estudian los alumnos de más bajos recursos. Contar con información sobre motivación de estos estudiantes complementaría muy bien los hallazgos discutidos. En segundo lugar, en este estudio no se contó con medidas de comprensión lectora, considerando su fuerte correlación con la motivación, habría sido interesante realizar ese análisis. Finalmente, el haber realizado algún tipo de entrevistas habría permitido contar con información cualitativa valiosa para interpretar estos resultados desde esa perspectiva. 


\section{Anexo. Ejemplo de ítems de MMRP}

Niño

2.

Un poco

1.

$\mathrm{Si}$
Niña

¿Te gusta leer libros, solo?

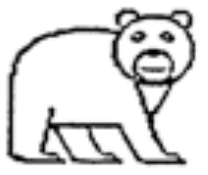

$$
\text { Un poco }
$$

3.

No

Aprender a leer es

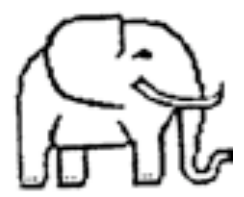

1.

No es muy

importante
2.

Es un poco

importante
3.

Es muy importante

\section{REFERENCIAS}

Afflerbach, P., Cho, B-Y, \& Kim, J. (2015). Conceptualizing and assessing higher order thinking in Reading. Theory into Practice, 54:3, 203-212.

Agencia de Calidad de la Educación (2017). Informe Nacional de resultados PIRLS 2016. Santiago, Chile. Disponible en http:/ / archivos.agenciaeducacion.cl/PIRLS_V02_27MAR.pdf

Allington, R.L. (2014). How Reading volume affects both Reading fluency and Reading achievement. International Electronic Journal of Elementary Education, 7(1), 13-26. 
Arango, P. (2018). Motivation: Introduction to the theory, concepts, and research. In P. Orellana y P. Baldwin (Eds.). Reading achievement and motivation in boys and girls. Field studies and methodological approaches. Dordrecht, Netherlands: Springer.

Brozo, W.G., Sulkunen, S., Shiel, G., Garbe, C., Pandian, A. \& Valtin, R. (2014) Journal of Adolescent y Adult Literacy, 57(7), 584-593

Cantrell, S. C., Rintamaa, M., Anderman, E. M., \& Anderman, L. H. (2017). Rural adolescents' reading motivation, achievement and behavior across transition to high school. The Journal of Educational Research, 1-12. doi:10.1080/00220671.2017.1284737

Case, R., \& Khanna, F. (1981). The missing links: Stages in children's progression from sensorimotor to logical thought. In K. W. Fischer (Ed.), Cognitive development: New directions for child development. San Francisco, CA: Jossey-Bassey.

Chall, J., \& Jacobs, V. (2003, Spring). The classic study on poor children's fourth-grade slump. American Educator, 27(1). Retrieved from http://www.aft.org/newspubs/periodicals/ae/ spring2003/hirschsbclassic.cfm

Collins, J. \& O'Brien, N. (2003.) The Greenwood dictionary of education. Westport, EE.UU.: Greenwood.

Conradi K., Jang B. G., McKenna M. C. (2014). Motivation terminology in reading research: a conceptual review. Educ. Psychol. Rev. 26 127-164. 10.1007/s10648-013-9245-z

Deci, E. L., \& Ryan, R. M. (2000). The "what" and "why" of goal pursuits: Human needs and the self-determination of behavior. Psychological Inquiry, 11(4), 227-268. doi: 10.1207/ S15327965PLI1104_01

Deci, E. L., \& Ryan, R. M. (2002). Self-determination research: Reflections and future directions. In E. L. Deci y R. M. Ryan (Eds.), Handbook of self-determination theory research (pp. 431-441). Rochester, NY: University of Rochester Press. 
Durik, A.M., Vida, M., \& Eccles, J.S. (2006). Task values and ability beliefs as predictors of high school literacy choices: A developmental analysis. Journal of Educational Psychology, 98(2), 382-393. doi:10.1037/0022-0663.98.2.382.

Fundación La Fuente / Adimark (2011). Estudio Chile y los Libros 2010. Santiago.

Gagné, M., \& Deci, E. L. (2005). Self-determination theory and work motivation. Journal of Organizational behavior, 26(4), 331-362.

Gambrell, L., Palmer, B., Codling, R., \& Mazzoni. S. (1996). Assessing motivation to read. The Reading Teacher, Vol. 49, No. 7 (Apr., 1996), pp. 518-533.

Gurian, M., Henley, P., \& Trueman, T. (2001). Boys and girls learn differently!: A guide for teachers and parents. San Francisco, CA, US: Jossey-Bass.

Guthrie, J. T., \& Wigfield, A. (2000). Engagement and motivation in reading. In P. B. M. M. L. Kamil, P. D. Pearson, y R. Barr (Ed.), Handbook of reading research, Vol. III (pp. 403-422). Mahway, NJ: Erlbaum.

Harkness, J. A., \& Schoua-Glusberg, A. (1998). Questionnaires in Translation. ZUMA-Nachrichten Spezial, Mannheim: ZUMA.

Kush, J.C., \& Watkins, M.W. (1996). Long-term stability of children's attitudes toward reading. The Journal of Educational Research, 89(5), 315-320.

Larson, L. (2010). Electronic reading workshop: Beyond books with new literacies and instructional technologies. Journal of Adolescent and Adult Literacy, 52, 121-131.

Lepper, M.R., Corpus, J.H. \& Iyengar, S.S. (2005). Intrinsic and extrinsic motivational orientations in the classroom: age differences and academic correlates. Journal of Educational Psychology, 97 (2), pp. 184-196. 
Ludwig, A. (2007). "The differences in the attitudes toward and perceptions of reading in successful and struggling middle school readers." (Master's thesis, Bowling Green).

Marinak, B. A., Malloy, J. B., Gambrell L. B., \& Mazzoni, S. A. (2015). Me and My Reading Profile: A Tool for Assessing Early Reading Motivation. The Reading Teacher, (69)1, 51-62.

Marinak, B. \& Gambrell, L. (2007). Boy's voices: I can read, I choose not to. Paper presented at the annual meeting of the College Reading Association, Salt Lake City, Utah, November 2, 2007.

Maynard, S. (2010). The Impact of e-Books on young children's reading habits. Publishing Research Quarterly, 26, 236248.10.1007/s12109-010-9180-5

McKenna, M.C., Conradi, K., Lawrence, C., Jang, B.G., \& Meyer, J. P. (2012). Reading attitudes of middle school students: Results of a U.S. survey. Reading Research Quarterly, 47(3), 283-306.

McKenna, M. C., Kear, D. J., \& Ellsworth, R. A. (1995). Children's attitudes toward reading: A national survey. Reading Research Quarterly, 30, 934-956.

Mol, S.E. \& Bus, A.G. (2011). To read or not to read: A meta-analysis of print exposure from infancy to early adulthood. Psychological Bulletin, 137(2), 267-296.

Morgan, P. L., Fuchs, D., Compton, D. L., Cordray, D. S., \& Fuchs, L. S. (2008). Does early reading failure decrease children's' reading motivation? Journal of Learning Disabilities, 41(5), 387-404.

Mullis, I.V.S., Martin, M.O., Foy, P., \& Drucker, K.T. (2012). International Results in Reading. Chestnut Hill, MA: TIMSS y PIRLS International Study Center, Boston College

Nitko, A. J. (1983). Educational tests and measurement: An introduction. New York, NY: Harcourt Brace Jovanovich. 
Orellana, P. y Baldwin, P. (2017). Motivación y desempeño lector en niñas y niños chilenos: un studio exploratorio. Revista Educationis Momentum, 2 (1), pp. 7-28

Organization for Economic Co-operation and Development. (2000). Literacy in the Information Age. Final Report of the International Adult Literacy Survey. OECD Publications Service: Paris, France: Autor.

OECD (2010). PISA 2009 Results: Learning to learn: Student engagement, Strategies and Practices (Volume III) OECD Publishing, Paris, http:/ / dx.doi.org/10.1787/9789264081925-en

Robb, L. (2011). The myth of learn to read/read to learn. Scholastic Instructor. Retrieved from http://www.scholastic.com/teachers/ article/myth-learn-readread-learn

Ryan, R. M., \& Deci, E. L. (2000). Self-determination theory and the facilitation of intrinsic motivation, social development, and well-being. American psychologist, 55(1), 68.

Schwanenflugel, P.J. \& Knapp, N.F. (2015). The psychology of reading: Theory and applications. New York: Guilford Press.

Wigfield, A., \& Eccles, J. S. (2000). Expectancy-value theory of motivation. Contemporary Educational Psychology, 25, 68-81.

Wigfield, A., Gladstone, J. R. \& Turci, L. (2016), Beyond Cognition: Reading Motivation and Reading Comprehension. Child Dev Perspect, 10: 190-195. doi:10.1111/cdep.12184 